\title{
Research Article \\ Impact of Common Property (E.A.) on Fixed Point Theorems in Fuzzy Metric Spaces
}

\author{
D. Gopal, ${ }^{1}$ M. Imdad, ${ }^{2}$ and C. Vetro ${ }^{3}$ \\ ${ }^{1}$ Department of Mathematics and Humanities, National Institute of Technology, Surat, Gujarat \\ 395007, India \\ 2 Department of Mathematics, Aligarh Muslim University, Aligarh 202002, India \\ ${ }^{3}$ Dipartimento di Matematica e Informatica, Università degli Studi di Palermo, Via Archirafi 34, \\ 90123 Palermo, Italy
}

Correspondence should be addressed to D. Gopal, gopal.dhananjay@rediffmail.com

Received 7 November 2010; Accepted 9 March 2011

Academic Editor: Jerzy Jezierski

Copyright (C) 2011 D. Gopal et al. This is an open access article distributed under the Creative Commons Attribution License, which permits unrestricted use, distribution, and reproduction in any medium, provided the original work is properly cited.

We observe that the notion of common property (E.A.) relaxes the required containment of range of one mapping into the range of other which is utilized to construct the sequence of joint iterates. As a consequence, a multitude of recent fixed point theorems of the existing literature are sharpened and enriched.

\section{Introduction and Preliminaries}

The evolution of fuzzy mathematics solely rests on the notion of fuzzy sets which was introduced by Zadeh [1] in 1965 with a view to represent the vagueness in everyday life. In mathematical programming, the problems are often expressed as optimizing some goal functions equipped with specific constraints suggested by some concrete practical situations. There exist many real-life problems that consider multiple objectives, and generally, it is very difficult to get a feasible solution that brings us to the optimum of all the objective functions. Thus, a feasible method of resolving such problems is the use of fuzzy sets [2]. In fact, the richness of applications has engineered the all round development of fuzzy mathematics. Then, the study of fuzzy metric spaces has been carried out in several ways (e.g., [3, 4]). George and Veeramani [5] modified the concept of fuzzy metric space introduced by Kramosil and Michálek [6] with a view to obtain a Hausdorff topology on fuzzy metric spaces, and this has recently found very fruitful applications in quantum particle physics, particularly in connection with both string and $\varepsilon^{\infty}$ theory (see [7] and references cited therein). In recent years, many authors have proved fixed point and common fixed point theorems in fuzzy metric spaces. To mention a few, we cite [2, 8-15]. As patterned 
in Jungck [16], a metrical common fixed point theorem generally involves conditions on commutatively, continuity, completeness together with a suitable condition on containment of ranges of involved mappings by an appropriate contraction condition. Thus, research in this domain is aimed at weakening one or more of these conditions. In this paper, we observe that the notion of common property (E.A.) relatively relaxes the required containment of the range of one mapping into the range of other which is utilized to construct the sequence of joint iterates. Consequently, we obtain some common fixed point theorems in fuzzy metric spaces which improve many known earlier results (e.g., $[11,15,17])$.

Before presenting our results, we collect relevant background material as follows.

Definition 1.1 (see [18]). Let $X$ be any set. A fuzzy set in $X$ is a function with domain $X$ and values in $[0,1]$.

Definition 1.2 (see [6]). A binary operation $*:[0,1] \times[0,1] \rightarrow[0,1]$ is a continuous $t$-norm if it satisfies the following conditions:

(i) $*$ is associative and commutative,

(ii) $*$ is continuous,

(iii) $a * 1=a$ for every $a \in[0,1]$,

(iv) $a * b \leq c * d$ if $a \leq c$ and $b \leq d$ for all $a, b, c, d \in[0,1]$.

Definition 1.3 (see [5]). A triplet $(X, M, *)$ is a fuzzy metric space whenever $X$ is an arbitrary set, $*$ is a continuous $t$-norm, and $M$ is a fuzzy set on $X \times X \times(0,+\infty)$ satisfying, for every $x, y, z \in X$ and $s, t>0$, the following conditions:

(i) $M(x, y, t)>0$,

(ii) $M(x, y, t)=1$ if and only if $x=y$,

(iii) $M(x, y, t)=M(y, x, t)$,

(iv) $M(x, y, t) * M(y, z, s) \leq M(x, z, t+s)$,

(v) $M(x, y, \cdot):(0,+\infty) \rightarrow[0,1]$ is continuous.

Note that $M(x, y, t)$ can be realized as the measure of nearness between $x$ and $y$ with respect to $t$. It is known that $M(x, y, \cdot)$ is nondecreasing for all $x, y \in X$. Let $(X, M, *)$ be a fuzzy metric space. For $t>0$, the open ball $B(x, r, t)$ with center $x \in X$ and radius $0<r<1$ is defined by $B(x, r, t)=\{y \in X: M(x, y, t)>1-r\}$. Now, the collection $\{B(x, r, t): x \in X$, $0<r<1, t>0\}$ is a neighborhood system for a topology $\tau$ on $X$ induced by the fuzzy metric $M$. This topology is Hausdorff and first countable.

Definition 1.4 (see [5]). A sequence $\left\{x_{n}\right\}$ in $X$ converges to $x$ if and only if for each $\varepsilon>0$ and each $t>0$, there exists $n_{0} \in N$ such that $M\left(x_{n}, x, t\right)>1-\varepsilon$ for all $n \geq n_{0}$.

Remark 1.5 (see [5]). Let $(X, d)$ be a metric space. We define $a * b=a b$ for all $a, b \in[0,1]$ and $M_{d}(x, y, t)=t /(t+d(x, y))$ for every $(x, y, t) \in X \times X \times(0,+\infty)$, then $\left(X, M_{d}, *\right)$ is a fuzzy metric space. The fuzzy metric space $\left(X, M_{d}, *\right)$ is complete if and only if the metric space $(X, d)$ is complete.

With a view to accommodate a wider class of mappings in the context of common fixed point theorems, Sessa [19] introduced the notion of weakly commuting mappings which was 
further enlarged by Jungck [20] by defining compatible mappings. After this, there came a host of such definitions which are scattered throughout the recent literature whose survey and illustration (up to 2001) is available in Murthy [21]. Here, we enlist the only those weak commutatively conditions which are relevant to presentation.

Definition 1.6 (see [20]). A pair of self-mappings $(f, g)$ defined on a fuzzy metric space $(X, M, *)$ is said to be compatible (or asymptotically commuting) if for all $t>0$,

$$
\lim _{n \rightarrow+\infty} M\left(f g x_{n}, g f x_{n}, t\right)=1,
$$

whenever $\left\{x_{n}\right\}$ is a sequence in $X$ such that $\lim _{n \rightarrow+\infty} f x_{n}=\lim _{n \rightarrow+\infty} g x_{n}=z$, for some $z \in X$. Also, the pair $(f, g)$ is called noncompatible, if there exists a sequence $\left\{x_{n}\right\}$ in $X$ such that $\lim _{n \rightarrow+\infty} f x_{n}=\lim _{n \rightarrow+\infty} g x_{n}=z$, but either $\lim _{n \rightarrow+\infty} M\left(f g x_{n}, g f x_{n}, t\right) \neq 1$ or the limit does not exist.

Definition 1.7 (see [10]). A pair of self-mappings $(f, g)$ defined on a fuzzy metric space $(X, M, *)$ is said to satisfy the property (E.A.) if there exists a sequence $\left\{x_{n}\right\}$ in $X$ such that $\lim _{n \rightarrow+\infty} f x_{n}=\lim _{n \rightarrow+\infty} g x_{n}=z$ for some $z \in X$.

Clearly, compatible as well as noncompatible pairs satisfy the property (E.A.).

Definition 1.8 (see [10]). Two pairs of self mappings $(A, S)$ and $(B, T)$ defined on a fuzzy metric space $(X, M, *)$ are said to share common property (E.A.) if there exist sequences $\left\{x_{n}\right\}$ and $\left\{y_{n}\right\}$ in $X$ such that $\lim _{n \rightarrow+\infty} A x_{n}=\lim _{n \rightarrow+\infty} S x_{n}=\lim _{n \rightarrow+\infty} B y_{n}=\lim _{n \rightarrow+\infty} T y_{n}=z$ for some $z \in X$.

For more on properties (E.A.) and common (E.A.), one can consult [22] and [10], respectively.

Definition 1.9. Two self mappings $f$ and $g$ on a fuzzy metric space $(X, M, *)$ are called weakly compatible if they commute at their point of coincidence; that is, $f x=g x$ implies $f g x=g f x$.

Definition 1.10 (see [23]). Two finite families of self mappings $\left\{A_{i}\right\}$ and $\left\{B_{j}\right\}$ are said to be pairwise commuting if

(i) $A_{i} A_{j}=A_{j} A_{i}, i, j \in\{1,2, \ldots, m\}$,

(ii) $B_{i} B_{j}=B_{j} B_{i}, i, j \in\{1,2, \ldots, n\}$,

(iii) $A_{i} B_{j}=B_{j} A_{i}, i \in\{1,2, \ldots, m\}$ and $j \in\{1,2, \ldots, n\}$,

The following definitions will be utilized to state various results in Section 3.

Definition 1.11 (see [15]). Let $(X, M, *)$ be a fuzzy metric space and $f, g: X \rightarrow X$ a pair of mappings. The mapping $f$ is called a fuzzy contraction with respect to $g$ if there exists an upper semicontinuous function $r:[0,+\infty) \rightarrow[0,+\infty)$ with $r(\tau)<\tau$ for every $\tau>0$ such that

$$
\frac{1}{M(f x, f y, t)}-1 \leq r\left(\frac{1}{m(f, g, x, y, t)}-1\right)
$$


for every $x, y \in X$ and each $t>0$, where

$$
m(f, g, x, y, t)=\min \{M(g x, g y, t), M(f x, g x, t), M(f y, g y, t)\} .
$$

Definition 1.12 (see [15]). Let $(X, M, *)$ be a fuzzy metric space and $f, g: X \rightarrow X$ a pair of mappings. The mapping $f$ is called a fuzzy $k$-contraction with respect to $g$ if there exists $k \in(0,1)$, such that

$$
\frac{1}{M(f x, f y, t)}-1 \leq k\left(\frac{1}{m(f, g, x, y, t)}-1\right)
$$

for every $x, y \in X$ and each $t>0$, where

$$
m(f, g, x, y, t)=\min \{M(g x, g y, t), M(f x, g x, t), M(f y, g y, t)\} .
$$

Definition 1.13. Let $A, B, S$ and $T$ be four self mappings of a fuzzy metric space $(X, M, *)$. Then, the mappings $A$ and $B$ are called a generalized fuzzy contraction with respect to $S$ and $T$ if there exists an upper semicontinuous function $r:[0,+\infty) \rightarrow[0,+\infty)$, with $r(\tau)<\tau$ for every $\tau>0$ such that for each $x, y \in X$ and $t>0$,

$$
\frac{1}{M(A x, B y, t)}-1 \leq r\left(\frac{1}{\min \{M(S x, T y, t), M(A x, S x, t), M(B y, T y, t)\}}-1\right) .
$$

\section{Main Results}

Now, we state and prove our main theorem as follows.

Theorem 2.1. Let $A, B, S$ and $T$ be self mappings of a fuzzy metric space $(X, M, *)$ such that the mappings $A$ and $B$ are a generalized fuzzy contraction with respect to mappings $S$ and $T$. Suppose that the pairs $(A, S)$ and $(B, T)$ share the common property (E.A.) and $S(X)$ and $T(X)$ are closed subsets of $X$. Then, the pair $(A, S)$ as well as $(B, T)$ have a point of coincidence each. Further, $A, B, S$ and $T$ have a unique common fixed point provided that both the pairs $(A, S)$ and $(B, T)$ are weakly compatible.

Proof. Since the pairs $(A, S)$ and $(B, T)$ share the common property (E.A.), there exist sequences $\left\{x_{n}\right\}$ and $\left\{y_{n}\right\}$ in $X$ such that for some $z \in X$,

$$
\lim _{n \rightarrow+\infty} A x_{n}=\lim _{n \rightarrow+\infty} S x_{n}=\lim _{n \rightarrow+\infty} B y_{n}=\lim _{n \rightarrow+\infty} T y_{n}=z
$$

Since $S(X)$ is a closed subset of $X$, therefore $\lim _{n \rightarrow+\infty} S x_{n}=z \in S(X)$, and henceforth, there exists a point $u \in X$ such that $S u=z$.

Now, we assert that $A u=S u$. If not, then by (1.6), we have

$$
\frac{1}{M\left(A u, B y_{n}, t\right)}-1 \leq r\left(\frac{1}{\min \left\{M\left(S u, T y_{n}, t\right), M(A u, S u, t), M\left(B y_{n}, T y_{n}, t\right)\right\}}-1\right),
$$


which on making $n \rightarrow+\infty$, for every $t>0$, reduces to

$$
\frac{1}{M(A u, z, t)}-1 \leq r\left(\frac{1}{\min \{M(A u, z, t)\}}-1\right)
$$

that is a contradiction yielding thereby $A u=S u$. Therefore, $u$ is a coincidence point of the pair $(A, S)$.

If $T(X)$ is a closed subset of $X$, then $\lim _{n \rightarrow+\infty} T y_{n}=z \in T(X)$. Therefore, there exists a point $w \in X$ such that $T w=z$.

Now, we assert that $B w=T w$. If not, then according to (1.6), we have

$$
\frac{1}{M\left(A x_{n}, B w, t\right)}-1 \leq r\left(\frac{1}{\min \left\{M\left(S x_{n}, T w, t\right), M\left(A x_{n}, S x_{n}, t\right), M(B w, T w, t)\right\}}-1\right),
$$

which on making $n \rightarrow+\infty$, for every $t>0$, reduces to

$$
\frac{1}{M(z, B w, t)}-1 \leq r\left(\frac{1}{\min \{M(z, B w, t)\}}-1\right)
$$

which is a contradiction as earlier. It follows that $B w=T w$ which shows that $w$ is a point of coincidence of the pair $(B, T)$. Since the pair $(A, S)$ is weakly compatible and $A u=S u$, hence $A z=A S u=S A u=S z$.

Now, we assert that $z$ is a common fixed point of the pair $(A, S)$. Suppose that $A z \neq z$, then using again (1.6), we have for all $t>0$,

$$
\frac{1}{M(A z, B w, t)}-1 \leq r\left(\frac{1}{\min \{M(A z, B w, t)\}}-1\right),
$$

implying thereby that $A z=B w=z$.

Finally, using the notion of weak compatibility of the pair $(B, T)$ together with (1.6), we get $B z=z=T z$. Hence, $z$ is a common fixed point of both the pairs $(A, S)$ and $(B, T)$.

Uniqueness of the common fixed point $z$ is an easy consequence of condition (1.6).

The following example is utilized to highlight the utility of Theorem 2.1 over earlier relevant results.

Example 2.2. Let $X=[2,20]$ and $(X, M, *)$ be a fuzzy metric space defined as

$$
M(x, y, t)=\frac{t}{t+|x-y|} \quad \text { if } t>0, x, y \in X
$$


Define $A, B, S, T: X \rightarrow X$ by

$$
\begin{gathered}
A x=\left\{\begin{array}{ll}
2 & \text { if } x=2, \\
3 & \text { if } x>2,
\end{array} \quad S x= \begin{cases}2 & \text { if } x=2, \\
6 & \text { if } x>2,\end{cases} \right. \\
B x=\left\{\begin{array}{ll}
2 & \text { if } x=2, \\
6 & \text { if } 2<x \leq 5, \\
3 & \text { if } x>5,
\end{array} \quad T x= \begin{cases}2 & \text { if } x=2, \\
18 & \text { if } 2<x \leq 5, \\
12 & \text { if } x>5 .\end{cases} \right.
\end{gathered}
$$

Then, $A, B, S$ and $T$ satisfy all the conditions of the Theorem 2.1 with $r(\tau)=k \tau$, where $k \in(4 / 9,1)$ and have a unique common fixed point $x=2$ which also remains a point of discontinuity.

Moreover, it can be seen that $A(X)=\{2,3\} \not \subset\{2,12,18\}=T(X)$ and $B(X)=$ $\{2,3,6\} \not \subset\{2,6\}=S(X)$. Here, it is worth noting that none of the earlier theorems (with rare possible exceptions) can be used in the context of this example as most of earlier theorems require conditions on the containment of range of one mapping into the range of other.

In the foregoing theorem, if we set $r(\tau)=k \tau, k \in(0,1)$, and $M(x, y, t)=t /(t+|x-y|)$, then we get the following result which improves and generalizes the result of Jungck [16, Corollary 3.2] in metric space.

Corollary 2.3. Let $A, B, S$ and $T$ be self mappings of a metric space $(X, d)$ such that

$$
d(A x, B y) \leq k \max \{d(S x, T y), d(A x, S x), d(B y, T y)\},
$$

for every $x, y \in X, k \in(0,1)$. Suppose that the pairs $(A, S)$ and $(B, T)$ share the common property (E.A.) and $S(X)$ and $T(X)$ are closed subsets of $X$. Then, the pair $(A, S)$ as well as $(B, T)$ have a point of coincidence each. Further, $A, B, S$ and $T$ have a unique common fixed point provided that both the pairs $(A, S)$ and $(B, T)$ are weakly compatible.

By choosing $A, B, S$ and $T$ suitably, one can deduce corollaries for a pair as well as for two different trios of mappings. For the sake of brevity, we deduce, by setting $A=B$ and $S=T$, a corollary for a pair of mappings which is an improvement over the result of C. Vetro and P. Vetro [15, Theorem 2].

Corollary 2.4. Let $(A, S)$ be a pair of self mappings of a fuzzy metric space $(X, M, *)$ such that $(A, S)$ satisfies the property (E.A.), $A$ is a fuzzy contraction with respect to $S$ and $S(X)$ is a closed subset of $X$. Then, the pair $(A, S)$ has a point of coincidence, whereas the pair $(A, S)$ has a unique common fixed point provided that it is weakly compatible.

Now, we know that $A$ fuzzy $k$-contraction with respect to $S$ implies $A$ fuzzy contraction with respect to $S$. Thus, we get the following corollary which sharpen of $[15$, Theorem 4 ].

Corollary 2.5. Let $A$ and $S$ be self mappings of a fuzzy metric space $(X, M, *)$ such that the pair $(A, S)$ enjoys the property (E.A.), $A$ is a fuzzy $k$-contraction with respect to $S$, and $S(X)$ is a closed 
Fixed Point Theory and Applications

subset of $X$. Then, the pair $(A, S)$ has a point of coincidence. Further, $A$ and $S$ have a unique common fixed point provided that the pair $(A, S)$ is weakly compatible.

\section{Implicit Functions and Common Fixed Point}

We recall the following two implicit functions defined and studied in [14] and [23], respectively.

Firstly, following Singh and Jain [14], let $\Phi$ be the set of all real continuous functions $\phi:[0,1]^{4} \rightarrow \mathbb{R}$, non decreasing in first argument, and satisfying the following conditions:

(i) for $u, v \geq 0, \phi(u, v, u, v) \geq 0$, or $\phi(u, v, v, u) \geq 0$ implies that $u \geq v$,

(ii) $\phi(u, u, 1,1) \geq 0$ implies that $u \geq 1$.

Example 3.1. Define $\phi\left(t_{1}, t_{2}, t_{3}, t_{4}\right)=15 t_{1}-13 t_{2}+5 t_{3}-7 t_{4}$. Then, $\phi \in \Phi$.

Secondly, following Imdad and Ali [23], let $\Psi$ denote the family of all continuous functions $F:[0,1]^{4} \rightarrow \mathbb{R}$ satisfying the following conditions:

(i) $F_{1}$ : for every $u>0, v \geq 0$ with $F(u, v, u, v) \geq 0$ or $F(u, v, v, u) \geq 0$, we have $u>v$,

(ii) $F_{2}: F(u, u, 1,1)<0$, for each $0<u<1$.

The following examples of functions $F \in \Psi$ are essentially contained in [23].

Example 3.2. Define $F:[0,1]^{4} \rightarrow \mathbb{R}$ as $F\left(t_{1}, t_{2}, t_{3}, t_{4}\right)=t_{1}-\phi\left(\min \left\{t_{2}, t_{3}, t_{4}\right\}\right)$, where $\phi:[0,1] \rightarrow$ $[0,1]$ is a continuous function such that $\phi(s)>s$ for $0<s<1$.

Example 3.3. Define $F:[0,1]^{4} \rightarrow \mathbb{R}$ as $F\left(t_{1}, t_{2}, t_{3}, t_{4}\right)=t_{1}-k \min \left\{t_{2}, t_{3}, t_{4}\right\}$, where $k>1$.

Example 3.4. Define $F:[0,1]^{4} \rightarrow \mathbb{R}$ as $F\left(t_{1}, t_{2}, t_{3}, t_{4}\right)=t_{1}-k t_{2}-\min \left\{t_{3}, t_{4}\right\}$, where $k>0$.

Example 3.5. Define $F:[0,1]^{4} \rightarrow \mathbb{R}$ as $F\left(t_{1}, t_{2}, t_{3}, t_{4}\right)=t_{1}-a t_{2}-b t_{3}-c t_{4}$, where $a>1$ and $b, c \geq 0(b, c \neq 1)$.

Example 3.6. Define $F:[0,1]^{4} \rightarrow \mathbb{R}$ as $F\left(t_{1}, t_{2}, t_{3}, t_{4}\right)=t_{1}-a t_{2}-b\left(t_{3}+t_{4}\right)$, where $a>1$ and $0 \leq b<1$.

Example 3.7. Define $F:[0,1]^{4} \rightarrow \mathbb{R}$ as $F\left(t_{1}, t_{2}, t_{3}, t_{4}\right)=t_{1}^{3}-k t_{2} t_{3} t_{4}$, where $k>1$.

Before proving our results, it may be noted that above-mentioned classes of functions $\Phi$ and $\Psi$ are independent classes as the implicit function $F\left(t_{1}, t_{2}, t_{3}, t_{4}\right)=t_{1}-k \min \left\{t_{2}, t_{3}, t_{4}\right\}$, where $k>1$ (belonging to $\Psi$ ) does not belongs to $\Phi$ as $F(u, u, 1,1)<0$ for all $u>0$, whereas implicit function $\phi\left(t_{1}, t_{2}, t_{3}, t_{4}\right)=15 t_{1}-13 t_{2}+5 t_{3}-7 t_{4}$ (belonging to $\Phi$ ) does not belongs to $\Psi$ as $F(u, v, u, v)=0$ implies $u=v$ instead of $u>v$. (E.A.).

The following lemma interrelates the property (E.A.) with the common property

Lemma 3.8. Let $A, B, S$ and $T$ be self mappings of a fuzzy metric space $(X, M, *)$. Assume that there exists $F \in \Psi$ such that

$$
F(M(A x, B y, t), M(S x, T y, t), M(S x, A x, t), M(B y, T y, t)) \geq 0,
$$


for all $x, y \in X$ and $t>0$. Suppose that pair $(A, S)$ (or $(B, T)$ ) satisfies the property (E.A.), and $A(X) \subset T(X)$ (or $B(X) \subset S(X)$ ). If for each $\left\{x_{n}\right\},\left\{y_{n}\right\}$ in $X$ such that $\lim _{n \rightarrow+\infty} A x_{n}=$ $\lim _{n \rightarrow+\infty} S x_{n}$ (or $\left.\lim _{n \rightarrow+\infty} B y_{n}=\lim _{n \rightarrow+\infty} T y_{n}\right)$, we have $\liminf _{n \rightarrow+\infty} M\left(A x_{n}, B y_{n}, t\right)>0$ for all $t>0$, then, the pairs $(A, S)$ and $(B, T)$ share the common property (E.A.).

Proof. If the pair $(A, S)$ enjoys the property (E.A.), then there exists a sequence $\left\{x_{n}\right\}$ in $X$ such that $\lim _{n \rightarrow+\infty} A x_{n}=\lim _{n \rightarrow+\infty} S x_{n}=z$ for some $z \in X$. Since $A(X) \subset T(X)$, hence for each $x_{n}$ there exists $y_{n}$ in $X$ such that $A x_{n}=T y_{n}$, henceforth $\lim _{n \rightarrow+\infty} A x_{n}=\lim _{n \rightarrow+\infty} T y_{n}=z$. Thus, we have $A x_{n} \rightarrow z, S x_{n} \rightarrow z$ and $T y_{n} \rightarrow z$.

Now, we assert that $B y_{n} \rightarrow z$. We note that $B y_{n} \rightarrow z$ if and only if $M\left(A x_{n}, B y_{n}, t\right) \rightarrow$ 1. Assume that there exists $t_{0}>0$ such that $M\left(A x_{n}, B y_{n}, t_{0}\right) \nrightarrow 1$, then by hypothesis there exists a subsequence of $\left\{x_{n}\right\}$, say $\left\{x_{n_{k}}\right\}$, such that

$$
M\left(A x_{n_{k}}, B y_{n_{k}}, t_{0}\right) \rightarrow \liminf _{n \rightarrow+\infty} M\left(A x_{n}, B y_{n}, t_{0}\right)=u>0
$$

By (3.1), we have

$$
F\left(M\left(A x_{n_{k}}, B y_{n_{k}}, t\right), M\left(S x_{n_{k}}, T y_{n_{k}}, t\right), M\left(S x_{n_{k}}, A x_{n_{k}}, t\right), M\left(B y_{n_{k}}, T y_{n_{k}}, t\right)\right) \geq 0,
$$

which on making $k \rightarrow+\infty$, reduces to

$$
F(u, 1,1, u) \geq 0
$$

implying thereby that $u>1$, which is a contradiction. Hence $\lim _{n \rightarrow+\infty} B y_{n}=z$ which shows that the pairs $(A, S)$ and $(B, T)$ share the common property (E.A.).

With a view to generalize some fixed point theorems contained in Imdad and Ali [11, 23] we prove the following fixed point theorem which in turn generalizes several previously known results due to Chugh and Kumar [24], Turkoglu et al. [25], Vasuki [18], and some others.

Theorem 3.9. Let $A, B, S$ and $T$ be self mappings of a fuzzy metric space $(X, M, *)$. Assume that there exists $F \in \Psi$ such that

$$
F(M(A x, B y, t), M(S x, T y, t), M(S x, A x, t), M(B y, T y, t)) \geq 0
$$

for all $x, y \in X$ and $t>0$. Suppose that the pairs $(A, S)$ and $(B, T)$ share the common property (E.A.) and $S(X)$ and $T(X)$ are closed subsets of $X$. Then, the pair $(A, S)$ as well as $(B, T)$ have a point of coincidence each. Further, $A, B, S$ and $T$ have a unique common fixed point provided that both the pairs $(A, S)$ and $(B, T)$ are weakly compatible.

Proof. Since the pairs $(A, S)$ and $(B, T)$ share the common property (E.A.), then there exist two sequences $\left\{x_{n}\right\}$ and $\left\{y_{n}\right\}$ in $X$ such that

$$
\lim _{n \rightarrow+\infty} A x_{n}=\lim _{n \rightarrow+\infty} S x_{n}=\lim _{n \rightarrow+\infty} B y_{n}=\lim _{n \rightarrow+\infty} T y_{n}=z,
$$

for some $z \in X$. 
Since $S(X)$ is a closed subset of $X$, then $\lim _{n \rightarrow+\infty} S x_{n}=z \in S(X)$. Therefore, there exists a point $u \in X$ such that $S u=z$. Then, by (3.5) we have

$$
F\left(M\left(A u, B y_{n}, t\right), M\left(S u, T y_{n}, t\right), M(S u, A u, t), M\left(B y_{n}, T y_{n}, t\right)\right) \geq 0
$$

which on making $n \rightarrow+\infty$ reduces to

$$
F(M(A u, z, t), M(S u, z, t), M(S u, A u, t), M(z, z, t)) \geq 0,
$$

or, equivalently,

$$
F(M(A u, z, t), 1, M(A u, z, t), 1) \geq 0
$$

which gives $M(A u, z, t)=1$ for all $t>0$, that is, $A u=z$. Hence, $A u=S u$. Therefore, $u$ is a point of coincidence of the pair $(A, S)$.

Since $T(X)$ is a closed subset of $X$, then $\lim _{n \rightarrow+\infty} T y_{n}=z \in T(X)$. Therefore, there exists a point $w \in X$ such that $T w=z$. Now, we assert that $B w=z$. Indeed, again using (3.5), we have

$$
F\left(M\left(A x_{n}, B w, t\right), M\left(S x_{n}, T w, t\right), M\left(S x_{n}, A x_{n}, t\right), M(B w, z, t)\right) \geq 0 .
$$

On making $n \rightarrow+\infty$, this inequality reduces to

$$
F(M(z, B w, t), M(z, z, t), M(z, z, t), M(B w, z, t)) \geq 0,
$$

that is,

$$
F(M(z, B w, t), 1,1, M(z, B w, t)) \geq 0
$$

implying thereby that $M(z, B w, t)>1$, for all $t>0$. Hence $T w=B w=z$, which shows that $w$ is a point of coincidence of the pair $(B, T)$. Since the pair $(A, S)$ is weakly compatible and $A u=S u$, we deduce that $A z=A S u=S A u=S z$.

Now, we assert that $z$ is a common fixed point of the pair $(A, S)$. Using (3.5), we have

$$
F(M(A z, B w, t), M(S z, T w, t), M(S z, A z, t), M(B w, T w, t)) \geq 0
$$

that is $F(M(A z, z, t), M(A z, z, t), 1,1) \geq 0$. Hence, $M(A z, z, t)=1$ for all $t>0$ and therefore $A z=z$.

Now, using the notion of the weak compatibility of the pair $(B, T)$ and (3.5), we get $B z=z=T z$. Hence, $z$ is a common fixed point of both the pairs $(A, S)$ and $(B, T)$. Uniqueness of $z$ is an easy consequence of (3.5).

Example 3.10. In the setting of Example 2.2, retain the same mappings $A, B, S$ and $T$ and define $F:[0,1]^{4} \rightarrow \mathbb{R}$ as $F\left(t_{1}, t_{2}, t_{3}, t_{4}\right)=t_{1}-\phi\left(\min \left\{t_{2}, t_{3}, t_{4}\right\}\right)$ with $\phi(r)=\sqrt{r}$. 
Then, $A, B, S$ and $T$ satisfy all the conditions of Theorem 3.9 and have a unique common fixed point $x=2$ which also remains a point of discontinuity.

Further, we remark that Theorem 2 of Imdad and Ali [23] cannot be used in the context of this example, as the required conditions on containment in respect of ranges of the involved mappings are not satisfied.

Corollary 3.11. The conclusions of Theorem 3.9 remain true if (3.5) is replaced by one of the following conditions:

(i) $M(A x, B y, t) \geq \phi(\min \{M(S x, T y, t), M(S x, A x, t), M(B y, T y, t)\})$, where $\phi:[0,1] \rightarrow$ $[0,1]$ is a continuous function such that $\phi(s)>s$ for all $0<s<1$.

(ii) $M(A x, B y, t) \geq k(\min \{M(S x, T y, t), M(S x, A x, t), M(B y, T y, t)\})$, where $k>1$.

(iii) $M(A x, B y, t) \geq k M(S x, T y, t)+\min \{M(S x, A x, t), M(B y, T y, t)\})$, where $k>0$.

(iv) $M(A x, B y, t) \geq a M(S x, T y, t)+b M(S x, A x, t)+c M(B y, T y, t)$, where $a>1$ and $b, c \geq 0(b, c \neq 1)$.

(v) $M(A x, B y, t) \geq a M(S x, T y, t)+b[M(S x, A x, t)+M(B y, T y, t)]$, where $a>1$ and $0 \leq b<1$.

(vi) $M(A x, B y, t) \geq k M(S x, T y, t) M(S x, A x, t) M(B y, T y, t)$, where $k>1$.

Proof. The proof of various corollaries corresponding to contractive conditions (i)-(vi) follows from Theorem 3.9 and Examples 3.2-3.7.

Remark 3.12. Corollary 3.11 corresponding to condition (i) is a result due to Imdad and Ali [11], whereas Corollary 3.11 corresponding to various conditions presents a sharpened form of Corollary 2 of Imdad and Ali [23]. Similar to this corollary, one can also deduce generalized versions of certain results contained in $[17,18,24]$.

The following theorem generalizes a theorem contained in Singh and Jain [14].

Theorem 3.13. Let $A, B, S$ and $T$ be self mappings of a fuzzy metric space $(X, M, *)$. Assume that there exists $\phi \in \Phi$ such that

$$
\begin{aligned}
& \phi(M(A x, B y, k t), M(S x, T y, t), M(A x, S x, t), M(B y, T y, k t)) \geq 0, \\
& \phi(M(A x, B y, k t), M(S x, T y, t), M(A x, S x, k t), M(B y, T y, t)) \geq 0,
\end{aligned}
$$

for all $x, y \in X, k \in(0,1)$ and $t>0$. Suppose that the pairs $(A, S)$ and $(B, T)$ enjoy the common property (E.A.) and $S(X)$ and $T(X)$ are closed subsets of $X$. Then, the pairs $(A, S)$ and $(B, T)$ have a point of coincidence each. Further, $A, B, S$ and $T$ have a unique common fixed point provided that both the pairs $(A, S)$ and $(B, T)$ are weakly compatible.

Proof. The proof of this theorem can be completed on the lines of the proof of Theorem 3.9, hence details are omitted.

Example 3.14. In the setting of Example 2.2, we define $\phi\left(t_{1}, t_{2}, t_{3}, t_{4}\right)=15 t_{1}-13 t_{2}+5 t_{3}-7 t_{4}$, besides retaining the rest of the example as it stands.

Then, all the conditions of Theorem 3.13 with $k \in(1 / 4,1)$ are satisfied. 
Notice that 2 is the unique common fixed point of $A, B, S$ and $T$, but this example cannot be covered by Theorem 3.1 due to Singh and Jain [14] as $A(X)=\{2,3\} \not \subset\{2,12,18\}=$ $T(X)$ and $B(X)=\{2,3,6\} \not \subset\{2,6\}=S(X)$. This example cannot also be covered by Theorem 3.9 of this paper as $\phi(u, u, 1,1)=2(u-1)$ implies $\phi(1,1,1,1)=0$ which contradicts $F_{1}$.

Now, we state (without proof) the following result.

Theorem 3.15. Let $\left\{A_{1}, A_{2}, \ldots, A_{m}\right\},\left\{B_{1}, B_{2}, \ldots, B_{n}\right\},\left\{S_{1}, S_{2}, \ldots, S_{p}\right\}$, and $\left\{T_{1}, T_{2}, \ldots, T_{q}\right\}$ be four finite families of self mappings of a fuzzy metric space $(X, M, *)$ such that the mappings $A=$ $A_{1} A_{2} \cdots A_{m}, B=B_{1} B_{2} \cdots B_{n}, S=S_{1} S_{2} \cdots S_{p}$ and $T=T_{1} T_{2} \cdots T_{q}$ satisfy (3.5). Suppose that the pairs $(A, S)$ and $(B, T)$ share the common property (E.A.) and $S(X)$ as well as $T(X)$ are closed subsets of X. Then, the pairs $(A, S)$ and $(B, T)$ have a point of coincidence each. Further, provided the pairs of families $\left(\left\{A_{i}\right\},\left\{S_{k}\right\}\right)$ and $\left(\left\{B_{r}\right\},\left\{T_{t}\right\}\right)$ commute pairwise, where $i \in\{1, \ldots, m\}, k \in\{1, \ldots, n\}, r \in$ $\{1, \ldots, p\}$, and $t \in\{1, \ldots, q\}$, then $A_{i}, S_{k}, B_{r}$ and $T_{t}$ have a unique common fixed point.

Proof. The proof of this theorem can be completed on the lines of Theorem 3.1 due to Imdad et al. [26], hence details are avoided.

By setting $A=A_{1}=A_{2}=\cdots=A_{m}, B=B_{1}=B_{2}=\cdots=B_{n}, S=S_{1}=S_{2}=\cdots=S_{p}$ and $T=T_{1}=T_{2}=\cdots=T_{q}$ in Theorem 3.15, one can deduce the following result for certain iterates of mappings which is a partial generalization of Theorem 3.9.

Corollary 3.16. Let $A, B, S$ and $T$ be four self mappings of a fuzzy metric space $(X, M, *)$ such that $A^{m}, B^{n}, S^{p}$ and $T^{q}$ satisfy the condition (3.5). Suppose that the pairs $\left(A^{m}, S^{p}\right)$ and $\left(B^{n}, T^{q}\right)$ share the common property (E.A.) and $S^{p}(X)$ as well as $T^{q}(X)$ are closed subsets of $X$. Then, the pairs $\left(A^{m}, S^{p}\right)$ and $\left(B^{n}, T^{q}\right)$ have a point of coincidence each. Further, $A, B, S$ and $T$ have a unique common fixed point provided that the pairs $(A, S)$ and $(B, T)$ commute pairwise.

Remark 3.17. Results similar to Corollary 3.11 as well as Corollary 3.16 can be outlined in respect of Theorem 3.13, Theorem 3.15, and Corollary 3.16. But due to the repetition, details are avoided.

Now, we conclude this note by deriving the following results of integral type.

Corollary 3.18. Let $A, B, S$ and $T$ be four self mappings of a fuzzy metric space $(X, M, *)$. Assume that there exist a Lebesgue integrable function $\varphi: \mathbb{R} \rightarrow \mathbb{R}$ and a function $\phi:[0,1]^{4} \rightarrow \mathbb{R}$ such that

$$
\int_{0}^{\phi(u, 1, u, 1)} \varphi(s) d s \geq 0, \quad \int_{0}^{\phi(u, 1,1, u)} \varphi(s) d s \geq 0, \quad \text { or } \quad \int_{0}^{\phi(u, u, 1,1)} \varphi(s) d s \geq 0
$$

implies $u=1$. Suppose that the pairs $(A, S)$ and $(B, T)$ share the common property (E.A.) and $S(X)$ and $T(X)$ are closed subsets of $X$. If

$$
\int_{0}^{\phi(M(A x, B y, t), M(S x, T y, t), M(S x, A x, t), M(B y, T y, t))} \varphi(s) d s \geq 0 \quad \forall x, y \in X \text { and } t>0,
$$

then the pairs $(A, S)$ and $(B, T)$ have a point of coincidence each. Further, $A, B, S$ and $T$ have a unique common fixed point provided that both the pairs $(A, S)$ and $(B, T)$ are weakly compatible. 
Proof. Since the pairs $(A, S)$ and $(B, T)$ share the common property (E.A.), then there exist two sequences $\left\{x_{n}\right\}$ and $\left\{y_{n}\right\}$ in $X$ such that

$$
\lim _{n \rightarrow+\infty} A x_{n}=\lim _{n \rightarrow+\infty} S x_{n}=\lim _{n \rightarrow+\infty} B y_{n}=\lim _{n \rightarrow+\infty} T y_{n}=z,
$$

for some $z \in X$. Since $S(X)$ is a closed subset of $X$, then $\lim _{n \rightarrow+\infty} S x_{n}=z \in S(X)$. Therefore, there exists a point $u \in X$ such that $S u=z$. Now, we assert that $A u=S u$. Indeed, by (3.16), we have

$$
\int_{0}^{\phi\left(M\left(A u, B y_{n}, t\right), M\left(S u, T y_{n}, t\right), M(S u, A u, t), M\left(B y_{n}, T y_{n}, t\right)\right)} \varphi(s) d s \geq 0
$$

On making $n \rightarrow+\infty$, it reduces to

$$
\int_{0}^{\phi(M(A u, z, t), 1, M(z, A u, t), 1)} \varphi(s) d s \geq 0
$$

which implies $M(A u, z, t)=1$, and so $A u=z$.

Being $T(X)$ a closed subset of $X$, repeating the same argument, we deduce that there exists a point $w \in X$ such that $B w=T w$.

Since the pair $(A, S)$ is weakly compatible and $A u=S u$, we deduce that $A z=A S u=$ $S A u=S z$.

Now, we assert that $z$ is a common fixed point of the pair $(A, S)$. Using (3.16), with $x=z$ and $y=w$, we have

$$
\int_{0}^{\phi(M(A z, z, t), M(A z, z, t), 1,1)} \varphi(s) d s \geq 0
$$

that implies $M(A z, z, t)=1$. Hence $A z=z$. Similarly, we prove that $B z=T z=z$ and so $z$ is a common fixed point of $A, B, S$ and $T$. Uniqueness of $z$ is a consequence of condition (3.16).

Corollary 3.19. Let $A, B, S$ and $T$ be four self mappings of a fuzzy metric space $(X, M, *)$. Assume that there exist a Lebesgue integrable function $\varphi: \mathbb{R} \rightarrow \mathbb{R}_{+}$and a function $\phi:[0,1]^{4} \rightarrow \mathbb{R}$, where $\phi \in \Phi$, such that

$$
\begin{gathered}
\int_{0}^{\phi(M(A x, B y, t), M(S x, T y, t), M(S x, A x, t), M(B y, T y, t))} \varphi(s) d s \geq 0, \forall x, y \in X, \quad t>0, \\
\int_{0}^{\phi(u, u, 1,1)} \varphi(s) d s \geq 0, \quad \forall u \in(0,1) .
\end{gathered}
$$

Suppose that the pairs $(A, S)$ and $(B, T)$ enjoy the common property (E.A.) and $S(X)$ and $T(X)$ are closed subsets of $X$. Then, the pairs $(A, S)$ and $(B, T)$ have a point of coincidence each. Further, 
$A, B, S$ and $T$ have a unique common fixed point provided that both the pairs $(A, S)$ and $(B, T)$ are weakly compatible.

Proof. The proof is the same of Corollary 3.18, so details are omitted.

\section{Acknowledgment}

C. Vetro is supported by University of Palermo, Local University project R. S. ex $60 \%$. The authors are grateful to Professor Dorel Mihet for going through the manuscript and for useful suggestions.

\section{References}

[1] L. A. Zadeh, "Fuzzy sets," Information and Computation, vol. 8, pp. 338-353, 1965.

[2] D. Turkoglu and B. E. Rhoades, "A fixed fuzzy point for fuzzy mapping in complete metric spaces," Mathematical Communications, vol. 10, no. 2, pp. 115-121, 2005.

[3] Z. Deng, "Fuzzy pseudometric spaces," Journal of Mathematical Analysis and Applications, vol. 86, no. 1, pp. 74-95, 1982.

[4] O. Kaleva and S. Seikkala, "On fuzzy metric spaces," Fuzzy Sets and Systems, vol. 12, no. 3, pp. 215-229, 1984.

[5] A. George and P. Veeramani, "On some results of analysis for fuzzy metric spaces," Fuzzy Sets and Systems, vol. 90, no. 3, pp. 365-368, 1997.

[6] I. Kramosil and J. Michálek, "Fuzzy metrics and statistical metric spaces," Kybernetika, vol. 11, no. 5, pp. 336-344, 1975.

[7] M. S. El Naschie, "On a fuzzy khaler-like manifold which is consistent with two slit experiment," International Journal of Nonlinear Sciences and Numerical Simulation, vol. 6, pp. 95-98, 2005.

[8] D. Mihet, "A generalization of a contraction principle in probabilistic metric spaces (II)," International Journal of Mathematics and Mathematical Sciences, vol. 2005, pp. 729-736, 2005.

[9] D. Mihet, "Fixed point theorems in fuzzy metric spaces using property E.A.," Nonlinear Analysis, vol. 73, pp. 2184-2188, 2010.

[10] M. Abbas, I. Altun, and D. Gopal, "Common fixed point theorems for non compatible mappings in fuzzy metric spaces," Bulletin of Mathematical Analysis and Applications, vol. 1, no. 2, pp. 47-56, 2009.

[11] M. Imdad and J. Ali, "Some common fixed point theorems in fuzzy metric spaces," Mathematical Communications, vol. 11, no. 2, pp. 153-163, 2006.

[12] V. Pant, "Contractive conditions and common fixed points in fuzzy metric space," Journal of Fuzzy Mathematics, vol. 14, no. 2, pp. 267-272, 2006.

[13] B. Singh and M. S. Chauhan, "Common fixed points of compatible maps in fuzzy metric spaces," Fuzzy Sets and Systems, vol. 115, no. 3, pp. 471-475, 2000.

[14] B. Singh and S. Jain, "Semicompatibility and fixed point theorems in fuzzy metric space using implicit relation," International Journal of Mathematics and Mathematical Sciences, no. 16, pp. 2617-2629, 2005.

[15] C. Vetro and P. Vetro, "Common fixed points for discontinuous mappings in fuzzy metric spaces," Rendiconti del Circolo Matematico di Palermo, vol. 57, no. 2, pp. 295-303, 2008.

[16] G. Jungck, "Commuting mappings and fixed points," The American Mathematical Monthly, vol. 83, no. 4, pp. 261-263, 1976.

[17] B. Singh and S. Jain, "Weak-compatibility and fixed point theorems in fuzzy metric space," Ganita, vol. 56, no. 2, pp. 167-176, 2005.

[18] R. Vasuki, "Common fixed points for $R$-weakly commuting maps in fuzzy metric spaces," Indian Journal of Pure and Applied Mathematics, vol. 30, no. 4, pp. 419-423, 1999.

[19] S. Sessa, "On a weak commutativity condition of mappings in fixed point considerations," Publications de l'Institut Mathématique, vol. 32(46), pp. 149-153, 1982.

[20] G. Jungck, “Compatible mappings and common fixed points," International Journal of Mathematics and Mathematical Sciences, vol. 9, no. 4, pp. 771-779, 1986.

[21] P. P. Murthy, "Important tools and possible applications of metric fixed point theory," Nonlinear Analysis: Theory, Methods \& Applications, vol. 47, no. 5, pp. 3479-3490, 2001.

[22] M. Aamri and D. El Moutawakil, "Some new common fixed point theorems under strict contractive conditions," Journal of Mathematical Analysis and Applications, vol. 270, no. 1, pp. 181-188, 2002. 
[23] M. Imdad and J. Ali, "A general fixed point theorem in fuzzy metric spaces via an implicit function," Journal of Applied Mathematics \& Informatics, vol. 26, pp. 591-603, 2008.

[24] R. Chugh and S. Kumar, "Common fixed point theorem in fuzzy metric spaces," Bulletin of the Calcutta Mathematical Society, vol. 94, no. 1, pp. 17-22, 2002.

[25] D. Turkoglu, C. Alaca, Y. J. Cho, and C. Yildiz, "Common fixed point theorems in intuitionistic fuzzy metric spaces," Journal of Applied Mathematics \& Computing, vol. 22, no. 1-2, pp. 411-424, 2006.

[26] M. Imdad, J. Ali, and M. Tanveer, "Coincidence and common fixed point theorems for nonlinear contractions in Menger PM spaces," Chaos, Solitons E Fractals, vol. 42, no. 5, pp. 3121-3129, 2009. 